\title{
Specific Cell Targeting with Nanobody Conjugated Branched Gold Nanoparticles for Photothermal Therapy
}

\author{
Bieke Van de Broek, ${ }^{\dagger, \pm, *}$ Nick Devoogdt, ${ }^{\S, \perp}$ Antoine D’Hollander, ${ }^{\dagger}$ Hannah-Laura Gijs, ${ }^{\dagger}$ Karolien Jans, ${ }^{\dagger}$ \\ Liesbet Lagae, ${ }^{\dagger,}$ Serge Muyldermans, ${ }^{\S, \perp}$ Guido Maes, $^{\ddagger}$ and Gustaaf Borghs ${ }^{\dagger}$ \\ ${ }^{\dagger}$ imec, Bio-Nano Electronics, Functional Nanosystems, Kapeldreef 75, B-3001 Leuven, Belgium, ${ }^{\ddagger}$ Department of Chemistry, Quantum and Physical Chemistry, \\ Katholieke Universiteit Leuven, Celestijnenlaan 200F, B-3001 Leuven, Belgium, ${ }^{\S}$ Department of Cellular and Molecular Interactions, Vlaams Interuniversitair \\ Instituut voor Biotechnologie (VIB), Pleinlaan 2, B-1050 Brussel, Belgium, ${ }^{\perp}$ Laboratory of Cellular and Molecular Immunology, Vrije Universiteit Brussel, Pleinlaan 2 , \\ B-1050 Brussel, Belgium, and "Department of Physics, Laboratory of Solid State Physics and Magnetism, Katholieke Universiteit Leuven, Celestijnenlaan 200D, \\ B-3001 Leuven, Belgium
}

$\mathrm{N}$ owadays, the use of nanoparticles in medicine is one of the most important directions of nanotechnology. ${ }^{1}$ There is a great deal of interest in the development of nanomaterials for photothermal therapy, a minimally invasive selective treatment of cancer. ${ }^{2}$ Photothermal therapy utilizes nanoparticles that absorb light in the near-infrared (NIR) region, where tissue (hemoglobin and water) has the highest transmissivity. ${ }^{3-5}$ The nanoparticles convert the absorbed photons into thermal energy, causing tumor cell destruction. ${ }^{2}$ The strong optical absorption of gold nanostructures makes them attractive as photothermal agents for cancer therapy. ${ }^{6}$ Different types of gold nanoparticles (nanoshells, nanorods, nanocages) have already been evaluated for photothermal therapy. ${ }^{1,2,6-12}$ A new type of gold nanomaterial that strongly absorbs NIR irradiation is branched gold nanoparticles. ${ }^{13,14}$ Because of their irregular shape and their very high surface-to-volume ratio, heat production is drastically enhanced. Elongated or sharp nanoparticles appear to be much more efficient heaters than massive nanostructures because the incoming electric field penetrates more easily inside the thin nanostructures making the whole amount of gold matter involved in heating. ${ }^{15-17}$ This makes branched gold nanoparticles potential candidates as photothermal therapeutic agents.

Such applications only become effective when the gold nanostructures have the capability to selectively target cancer cells
ABSTRACT Branched gold nanoparticles are potential photothermal therapy agents because of their large absorption cross section in the near-infrared window. Upon laser irradiation they produce enough heat to destroy tumor cells. In this work, branched gold nanoparticles are biofunctionalized with nanobodies, the smallest fully functional antigen-binding fragments evolved from the variable domain, the VHH, of a camel heavy chain-only antibody. These nanobodies bind to the HER2 antigen which is highly expressed on breast and ovarian cancer cells. Flow cytometric analysis and dark field images of HER2 positive SKOV3 cells incubated with anti-HER2 conjugated branched gold nanoparticles show specific cell targeting. Laser irradiation studies reveal that HER2 positive SKOV3 cells exposed to the anti-HER2 targeted branched gold nanoparticles are destroyed after five minutes of laser treatment at $38 \mathrm{~W} / \mathrm{cm}^{2}$ using a $690 \mathrm{~nm}$ continuous wave laser. Starting from a nanoparticle optical density of 4, cell death is observed, whereas the control samples, nanoparticles with anti-PSA nanobodies, nanoparticles only, and laser only, do not show any cell death. These results suggest that this new type of bioconjugated branched gold nanoparticles are effective antigen-targeted photothermal therapeutic agents for cancer treatment.

KEYWORDS: branched gold nanoparticles · nanobody conjugation · specific cell targeting $\cdot$ photothermal therapy

without affecting the healthy ones. ${ }^{18}$ Achieving this specific targeting is particularly important when laser irradiation is used for medical applications. The laser power that is needed to achieve therapeutic effects in cancers can be reduced in order to not exceed the medical safety standards. ${ }^{2}$ To increase the photothermal effectiveness of gold nanostructures, their physicochemical properties have to be carefully controlled. These properties include size, shape, morphology, charge, and surface chemistry. ${ }^{5}$ Aside from these physicochemical properties of the nanoparticles, high specificity is generally introduced by taking advantage of the physiological differences
* Address correspondence to Bieke.Vandebroek@imec.be.

Received for review September 8, 2010 and accepted May 24, 2011.

Published online May 25, 2011 10.1021/nn1023363

C일 2011 American Chemical Society 
between malignant and normal cells. ${ }^{2}$ In general, nanoparticles may accumulate in the target tissues through two mechanisms, passive and active targeting. Passive targeting is the nonspecific accumulation of nanoparticles in the tumors owing to the unique properties of the tumor microenvironment. ${ }^{5,19}$ Tumors have a leaky vasculature allowing nanoparticles with a small size to pass through and concentrate in the tumor through the enhanced permeability and retention effect. ${ }^{20,21}$ The size of the nanoparticles should be small enough $(<100 \mathrm{~nm})$ to go through the vasculature pores but large enough $(>5 \mathrm{~nm})$ to avoid rapid clearance. ${ }^{5,22}$ As a result of these characteristics, the concentration of nanoparticles found in tumor tissue can be up to 100 times higher than those in normal tissue. ${ }^{19}$ Active targeting is ligand-directed, site-specific accumulation of nanoparticles in tumors owing to an increased expression of specific cell surface markers on cancer cells. 5,23,24 These markers can be recognized by specific ligands conjugated on the surface of the nanoparticles. ${ }^{5,19}$ Antibodies represent a class of commonly used ligands that can be easily conjugated to the surface of gold nanostructures. ${ }^{18}$ Molecular targeting of the nanoparticles to cancer cells via antibodies to their cell surface markers may further enhance the accumulation of the nanoparticles in tumors, prolong their retention, and potentially allow the use of lower nanoparticle dosages. ${ }^{23,24}$ Among the many known cell surface markers for targeting, human epidermal growth factor receptor 2 (HER2) has been identified as a promising target for antibody-based therapy. HER2 is a transmembrane receptor protein involved in the signal transduction pathways which control cell growth and differentiation. ${ }^{25}$ In tumors overexpressing this marker, roughly $10^{6} \mathrm{HER} 2$ receptors per cell may be present. ${ }^{26}$ HER2 has been used to target breast cancer cells because of its stable overexpression on $30 \%$ of breast cancers. ${ }^{27,28}$ Aside from breast cancer, also $25 \%$ of the ovarian cancer cells show an overexpression of the HER2 receptor. ${ }^{24,25,29,30}$

Nanoparticle size is not the only important factor in determining the efficiency of the targeted delivery of gold nanostructures. The size of the targeting ligand attached on the surface of the nanoparticle must also be considered. ${ }^{5}$ There is an increasing interest in using antibody fragments that retain high antigen binding specificity to improve tumor penetration. ${ }^{19}$ Minimizing the size of antigen-binding proteins to a single immunoglobulin domain with high affinity for a target antigen has been one of the major goals of antibody engineering over the past decade. ${ }^{31}$ Nanobodies are the smallest fully functional antigen-binding fragments derived from the variable domain $(\mathrm{VHH})$ of naturally occurring heavy-chain-only antibodies (HCAbs) present in species of the camelidae. ${ }^{19,32-35}$ Nanobodies are distinguished from other conventional antibody formats by their unique properties of size $(2.5 \mathrm{~nm}$ in diameter and $4 \mathrm{~nm}$ high), intrinsic stability, and ease of manufacture (recombinant production). ${ }^{31,36,37}$ Given their high affinity and specificity, the small size of nanobodies (15 kDa) makes them particularly suitable for targeting antigens in obstructed locations, such as tumors, where penetration into poorly vascularized tissue is crucial. ${ }^{38} \mathrm{~A}$ striking property of nanobodies is their high thermal and conformational stability. ${ }^{39}$ It has been shown that nanobodies retain $>80 \%$ of their binding activity after 1 week of incubation at $37^{\circ} \mathrm{C}$. In addition, nanobodies regain antigen-binding specificity after prolonged incubation at elevated temperatures in the hyperthermia range $\left(40-47^{\circ} \mathrm{C}\right)$ and even up to $90{ }^{\circ} \mathrm{C}$. This indicates that nanobodies can be useful for hyperthermia therapy since they are stable upon prolonged incubation with cells at $37{ }^{\circ} \mathrm{C}$ and at elevated temperatures relevant for therapeutic applications. Moreover, nanobodies have a faster blood clearance and an extremely low immunogenic response compared to the complete antibody. ${ }^{31,32}$ These properties open perspectives for the use of nanobodies as tumor-targeting vehicles for therapeutic purposes such as hyperthermic treatment of cancer. ${ }^{38}$

In this work, we demonstrate the potential use of branched gold nanoparticles with a strong optical absorption in the NIR window as photothermal therapy agents. We have developed a method to conjugate the nanoparticles with anti-HER2 nanobodies to actively target HER2-expressing SKOV3 carcinoma cells. The specificity of these conjugated nanoparticles is evaluated in vitro by flow cytometry and dark field microscopy. Moreover, specific nanoparticle-induced photothermal destruction of the cells upon laser irradiation is established. The minimal nanoparticle concentration to achieve cell destruction is determined. Control samples such as nonspecific nanoparticles, laser only, and nanoparticles only are evaluated upon cell death.

\section{RESULTS AND DISCUSSION}

Synthesis and Biofunctionalization of Branched Gold Nanoparticles with anti-HER2 and anti-PSA Nanobodies. The branched gold nanoparticles were synthesized by a procedure based on Hao et al. ${ }^{13}$ and subsequently characterized with UV-vis absorption spectroscopy and TEM. They show a plasmon absorption band at $628 \mathrm{~nm}$ (Figure 1) and an average size of $60.4 \pm 9.7 \mathrm{~nm}$ (Figure 1 inset). ${ }^{13}$ Further tuning of the LSPR band in the biological window is possible via electroless plating with gold. ${ }^{40}$ These particles were stabilized and chemically functionalized by an exchange reaction that replaces the initial citrate capping layer with the maleimide-PEO-disulfide. ${ }^{41}$ The incorporation of PEO units is necessary to stabilize the nanoparticles in physiological environments. ${ }^{10}$ The maleimide is a preactivated end group, which allows a site-directed 


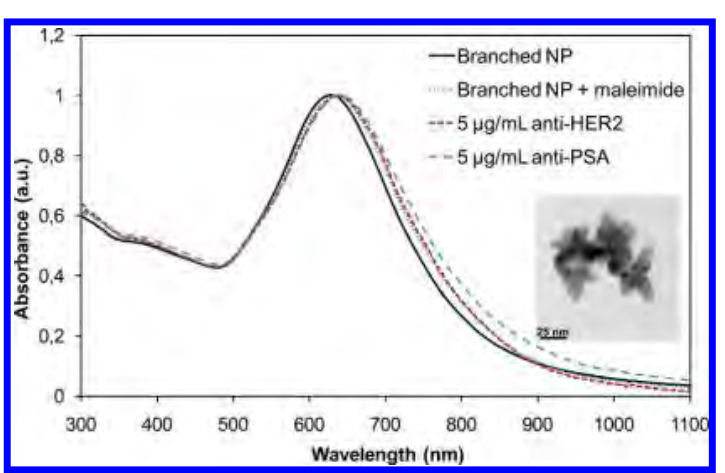

Figure 1. UV-vis absorption spectra of unmodified branched gold nanoparticles (plasmon absorption band at $628 \mathrm{~nm}$ ), branched gold nanoparticles chemically functionalized with the maleimide-PEO-disulfide (plasmon absorption band at $635 \mathrm{~nm}$ ), branched gold nanoparticles coupled with $5 \mu \mathrm{g} / \mathrm{mL}$ anti-HER2 nanobodies (plasmon absorption band at $637 \mathrm{~nm}$ ), and anti-PSA nanobodies (plasmon absorption band at $640 \mathrm{~nm}$ ). Inset: TEM image of unmodified branched gold nanoparticles (size: $60.4 \pm 9.7 \mathrm{~nm})$.

one-step reaction with free thiol groups present in the biomolecules. ${ }^{42,43}$ The resulting nanoparticles were characterized with UV-vis absorption spectroscopy. Their plasmon absorption band is shifted to $635 \mathrm{~nm}$ (Figure 1) and they have an optical density of 1 . With inductively coupled plasma-optical emission spectroscopy (ICP-OES) the concentration of these branched gold nanoparticles was determined. Using gold standards, a total gold concentration of $38.6 \mathrm{ppm}$ was measured for a sample of branched gold nanoparticles with OD 1 (see Supporting Information). The number of nanoparticles per milliliter was calculated by dividing this total gold concentration by the mass of gold in a single branched gold nanoparticle, which resulted in $2.3 \times 10^{10}$ $\mathrm{NP} / \mathrm{mL}$ for OD 1 (see Supporting Information).

In this study, anti-HER2 nanobodies and anti-PSA nanobodies (negative control) were conjugated to the branched gold nanoparticles to evaluate specific cell interactions. Hereto, the nanobodies were modified during production with a C-terminal cysteine which can form a thioether bond with the maleimide end group of the nanoparticle surface. To reduce possible binding of the $\mathrm{N}$-terminal amine group with the gold surface of the nanoparticles, a glutamate was inserted at the $\mathrm{N}$-terminal site. The carboxyl group of glutamate can cyclisize with the $\mathrm{N}$-terminal amine group to form a pyroglutamate, which prevents the amine group from binding directly to the gold nanoparticles.

The optimal concentration to couple the anti-HER2 nanobodies to the branched gold nanoparticles was determined in two steps. Hereto, both the nanoparticles and the nanobodies were dissolved in HEPES buffer (10 mM, pH 7.0). First, a broad concentration range of the nanobodies $(0.1,1,10$, and $100 \mu \mathrm{g} / \mathrm{mL})$ was used for coupling and evaluated with UV-vis absorption spectroscopy. The spectra show aggregation of the nanoparticles upon coupling of 10 and
$100 \mu \mathrm{g}$ of nanobodies $/ \mathrm{mL}$. For 0.1 and $1 \mu \mathrm{g} / \mathrm{mL}$ no aggregation was induced (see Supporting Information). As such, the optimal concentration to couple the nanobodies is situated between 1 and $10 \mu \mathrm{g} / \mathrm{mL}$. Therefore, a second concentration range $(0.625,1.25$, $2.5,5$, and $10 \mu \mathrm{g} / \mathrm{mL}$ ) was conducted, which shows that $5 \mu \mathrm{g} / \mathrm{mL}$ is the highest concentration of HER2 nanobodies that can be coupled to the nanoparticles without inducing aggregation (see Supporting Information). A similar optimization procedure was performed to couple the anti-PSA nanobodies to the branched gold nanoparticles. For this nanobody a higher concentration $(10 \mu \mathrm{g} / \mathrm{mL})$ could be coupled (see Supporting Information) but for further experiments $5 \mu \mathrm{g} / \mathrm{mL}$ of nanobodies was used to allow a good comparison with the anti-HER2 conjugated branched gold nanoparticles. Figure 1 shows the UV-vis absorption spectra for branched gold nanoparticles biofunctionalized with $5 \mu \mathrm{g} / \mathrm{mL}$ anti-HER2 and anti-PSA nanobodies, indicating a shift of their plasmon absorption band to 637 and $640 \mathrm{~nm}$, respectively.

Flow Cytometric and Dark Field Analysis of Specific CellNanoparticle Interactions. To further evaluate the effectiveness of the biofunctionalization of the branched gold nanoparticles with anti-HER2 nanobodies, their specific interaction with cells was evaluated. The free anti-HER2 nanobody has a high affinity to the HER2receptor, but it is important to determine whether the nanobodies still interact with the receptor after coupling to the nanoparticles. Two cell lines were used for this experiment, a human ovarian epithelial carcinoma cell line (SKOV3), which is HER2-positive, and a Chinese hamster ovary cell line (CHO), which is HER2-negative (see Supporting Information). ${ }^{44}$ The specific interaction of these nanobody-conjugated branched gold nanoparticles with HER2 + cells was evaluated in vitro with fluorescence activated cell sorting (FACS) using a fluorescent sandwich assay. The branched gold nanoparticles conjugated with the anti-HER2 nanobodies recognize the HER2 receptor on the cells. The nanobodies contain a hexahistidine-tag which is recognized by a primary anti-His monoclonal mouse $\operatorname{lgG}$. This primary antibody is recognized by a rat anti-mouse $\operatorname{lgG1}$, labeled with a fluorescent dye, phycoerythrin $(P E)$, which emits at $570 \mathrm{~nm}$. The signal of this second antibody is measured with FACS (Figure 2). For this fluorescent assay, one could question the possibility of quenching of the fluorescent signal due to the proximity of a gold nanoparticle near a fluorescent dye. Efficient quenching only occurs when several requirements are fulfilled. First, the dye should emit at a wavelength that is absorbed by the nanoparticle. In our case, there is only slight overlap between the emission of the dye $(570 \mathrm{~nm})$ and the absorption of the nanoparticles $(637 \mathrm{~nm})$. Second, quenching explicitly occurs when the distance between the nanoparticle and the fluorescent dye is within $10 \mathrm{~nm}$ and 


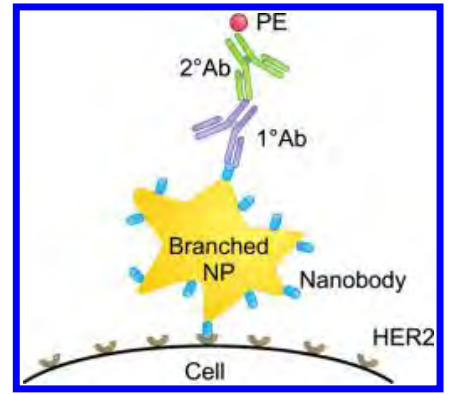

Figure 2. Schematic representation of the fluorescent assay used for the FACS experiment.

decreases significantly with increasing distance. ${ }^{45,46}$ In our assay, the distance is approximately $40 \mathrm{~nm}$, determined by the presence of the maleimide disulfide ( $5 \mathrm{~nm}$ ), two monoclonal antibodies ( $15 \mathrm{~nm}$ each), and a nanobody ( $5 \mathrm{~nm})$, and therefore quenching is less likely to occur. Finally, quenching decreases with increasing nanoparticle diameter, with a reduced quenching for $50 \mathrm{~nm}$ gold nanoparticles compared to $20 \mathrm{~nm}$ gold nanoparticles. ${ }^{46}$ Since our nanoparticles are about $60 \mathrm{~nm}$ in size, quenching is minimized as well in our case. These different aspects were taken into account when setting up the experiment and allowed us to have a significant fluorescent signal without too much quenching.

Both HER2 + SKOV3 cells and HER2- CHO cells were incubated with four different samples. The first sample contains the branched gold nanoparticles conjugated with the anti-HER2 nanobodies. The second sample is a positive control, free anti-HER2 nanobodies that are not coupled to the nanoparticles. The two last samples are negative controls, the branched gold nanoparticles with anti-PSA nanobodies and free anti-PSA nanobodies. After incubation, the cells were labeled with the primary anti-His monoclonal mouse $\lg \mathrm{G} 1$ and the secondary rat anti-mouse $\lg \mathrm{G} 1$ antibody. As a reference for all these samples, the cells were incubated with the primary and secondary antibody without nanoparticles or nanobodies. The FACS results are illustrated in Figure 3. The percentage of measured live cells is plotted versus the fluorescence signal intensity. A significant shift in fluorescence signal is observed when HER2 + SKOV3 cells are incubated with anti-HER2 conjugated nanoparticles compared to the cells without nanoparticles (Figure 3a). This indicates that these nanoparticles have a high affinity for the HER2 + cells and that the nanobodies do not lose their binding affinity upon conjugation to the branched gold nanoparticles. Although, not only do the nanobodies present on the nanoparticles that interact with the cells contribute to this fluorescence signal shift, but also the free nonbinding nanobodies on the nanoparticles contribute, which makes it difficult to determine the amount of cell-bound nanobodies. A slightly larger shift to higher intensities is observed for the interaction of the SKOV3 cells with the positive control, the antiHER2 nanobodies (Figure 3b). This signal comes from nanobodies that are all directly bound to the cells. This implies that the number of cell-bound free nanobodies is slightly larger than the number of nanobodies present on the cell-bound nanoparticles, but no conclusions can be drawn regarding the difference in affinity of the free nanobodies and the nanoparticle-bound nanobodies. The high specificity of these anti-HER2 conjugated branched gold nanoparticles is confirmed upon evaluation of the negative controls. Incubation of the HER2 + cells with the first negative control, antiPSA conjugated nanoparticles, only shows a low binding signal indicating a small amount of nonspecific binding to the cells (Figure 3c). The second negative control, free anti-PSA nanobodies, shows negligible binding to the HER2+ cells confirming that this nanobody has very low affinity for the HER2 receptor (Figure 3d). For the HER2- $\mathrm{CHO}$ cells (negative controls) the nanoparticles with anti-HER2 nanobodies show a limited shift in the fluorescence signal indicating a small amount of nonspecific binding to these cells. The anti-HER2 nanobodies alone show no increased fluorescence signal confirming that they do not bind to the HER2 - cells. For the anti-PSA nanobodies similar results are obtained: the anti-PSA conjugated nanoparticles show a low binding signal indicating a small amount of nonspecific binding and the free anti-PSA nanobodies have no affinity for the HER2 - cells. These results clearly prove the specific interaction of the anti-HER2 conjugated branched gold nanoparticles with HER2+ cells.

This specific interaction between the nanobodyfunctionalized nanoparticles and the cells was also confirmed using dark field microscopy. Hereto, the same samples were used as for the FACS analysis. For this type of microscopy, the light beam direction is optimized so that the center illumination beam does not enter the collection cone of the microscope objective. Only the scattered light from the sample is collected which presents an image of a bright object in a dark background. ${ }^{47}$ Figure 4 shows the light scattering images of the HER2+ SKOV3 cells and the HER2- CHO cells incubated with the four different samples. All the cells show white scattered light, which is due to autofluorescence and scattered light from the cell organelles. The branched gold nanoparticles scatter orange light due to their strong optical absorption at the onset of the NIR window. ${ }^{47}$ For the SKOV3 cells a strong interaction is observed with the anti-HER2 conjugated nanoparticles (Figure 4a). A high density of nanoparticles is clearly visible on the cells from the high amount of orange scattered light. This result confirms the specific binding of anti-HER2 conjugated nanoparticles as previously seen by the large shift in fluorescence intensity in the FACS analysis. Interaction with the anti-PSA 


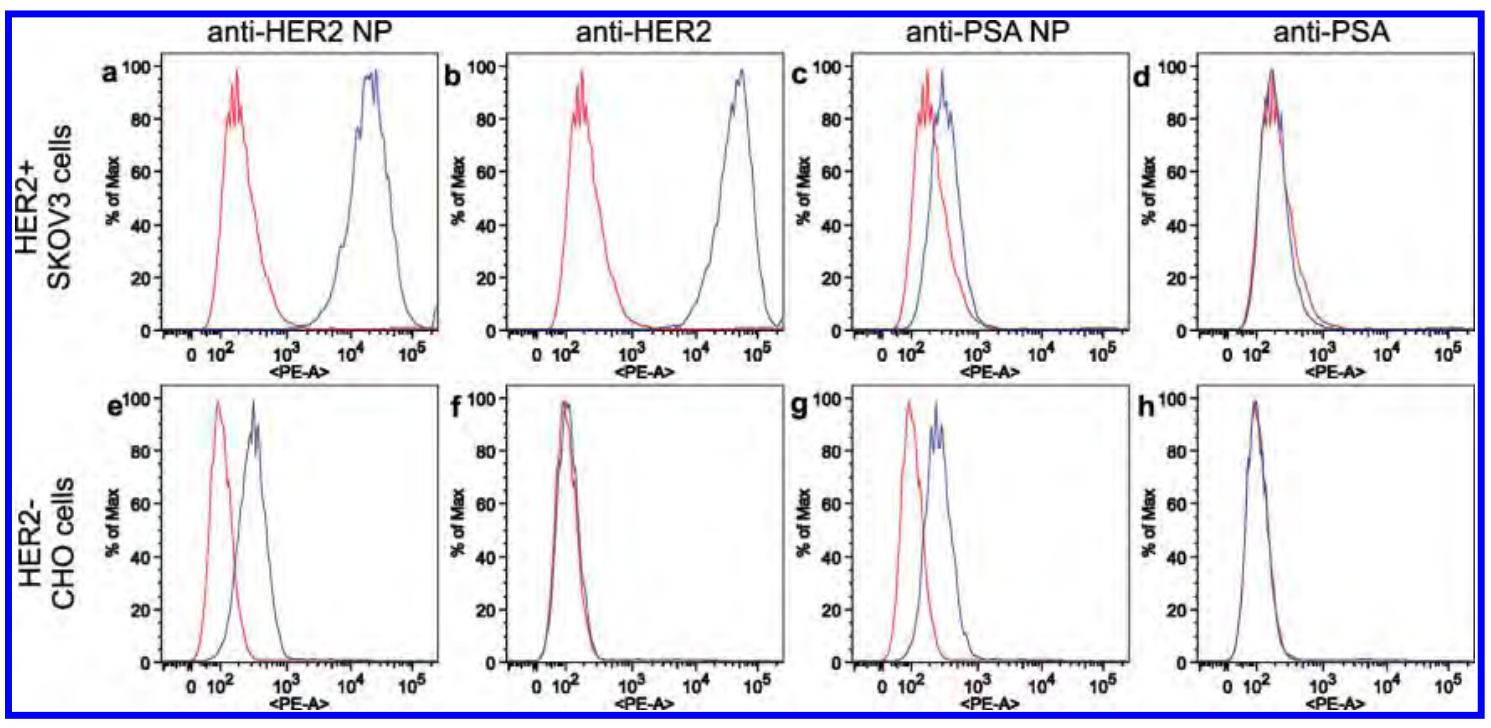

Figure 3. FACS analysis of SKOV3 cells incubated with (a) anti-HER2 NP, (b) anti-HER2 nanobodies (positive control), (c) anti-PSA NP (negative control), (d) anti-PSA nanobodies (negative control) and of CHO cells incubated with (e) anti-HER2 NP (negative control), (f) anti-HER2 nanobodies (negative control), (g) anti-PSA NP (negative control), (h) anti-PSA nanobodies (negative control) compared to the cells without NP or nanobody.

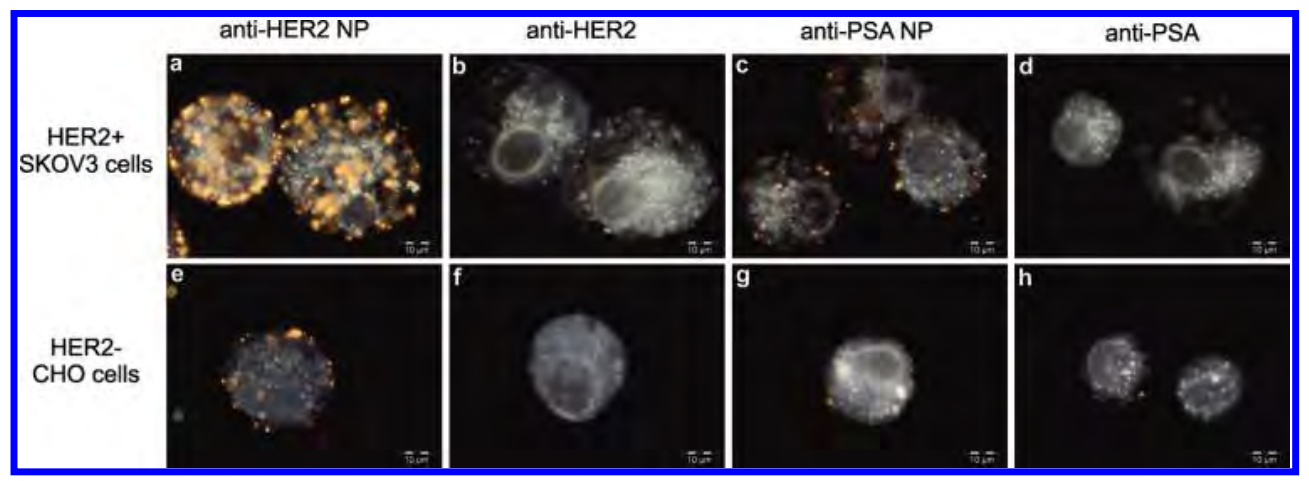

Figure 4. Dark field images of SKOV3 cells incubated with (a) anti-HER2 NP, (b) anti-HER2 nanobodies, (c) anti-PSA NP, (d) anti-PSA nanobodies and of CHO cells incubated with (e) anti-HER2 NP, (f) anti-HER2 nanobodies, (g) anti-PSA NP, (h) anti-PSA nanobodies.

conjugated nanoparticles only shows a limited binding as a small amount of orange scattering from the nanoparticles can be observed (Figure 4c). This is due to nonspecific binding that is also observed in the FACS analysis as a small shift in the fluorescence intensity. For the interaction with the free anti-HER2 (Figure 4b) and anti-PSA (Figure 4d) nanobodies no orange scattering is observed as no nanoparticles are added to the cells. For the $\mathrm{CHO}$ cells only a small amount of nonspecific binding is observed for the anti-HER2 conjugated nanoparticles (Figure 4e) as well as for the antiPSA conjugated nanoparticles (Figure $4 \mathrm{~g}$ ). This is in agreement with the small shift in fluorescence intensity observed in the FACS analysis. The interaction with the free nanobodies (Figure $4 \mathrm{f}, \mathrm{h}$ ) shows no orange light scattering as no nanoparticles are introduced.

From these FACS data and dark field images we conclude that the anti-HER2 conjugated nanoparticles show a highly specific interaction with the HER2+ SKOV3 cells. For the negative controls with free nanobodies (SKOV3 cells with anti-PSA, CHO cells with anti-HER2 and anti-PSA) no binding is observed. The negative controls with nonspecific nanoparticles (SKOV3 cells with anti-PSA NP, CHO cells with antiHER2 NP and anti-PSA NP) show a small amount of nonspecific binding, which is attributed to the small amount of free reactive maleimide groups that are still present on the nanoparticle surface. This nonspecific binding can be reduced by blocking these maleimide groups before incubation.

Photothermal Therapy of HER2 Positive Cells with Specific anti-HER2 Branched Gold Nanoparticles. Finally, the efficiency of these highly specific branched gold nanoparticles as photothermal agents to destroy the targeted cancer cells was evaluated in vitro. Hereto, SKOV3 cells were incubated with anti-HER2 conjugated branched gold nanoparticles with different concentrations (OD $6\left(1.4 \times 10^{11} \mathrm{NP} / \mathrm{mL}\right)$, OD $4\left(9.2 \times 10^{10} \mathrm{NP}\right.$ / $\mathrm{mL})$, OD $2\left(4.6 \times 10^{10} \mathrm{NP} / \mathrm{mL}\right)$, OD $\left.1\left(2.3 \times 10^{10} \mathrm{NP} / \mathrm{mL}\right)\right)$ for $1 \mathrm{~h}$. After washing three times with PBS, the cells 


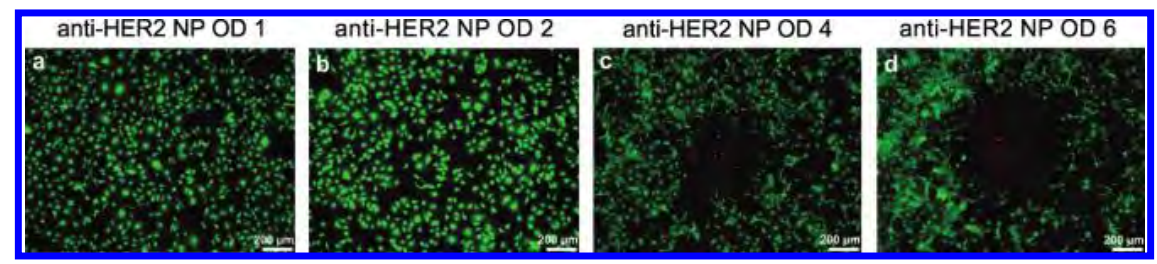

Figure 5. SKOV3 cells incubated with anti-HER2 conjugated branched gold nanoparticles at different concentrations (a) OD 1, (b) OD 2, (c) OD 4, (d) OD 6 after laser irradiation and live/dead staining.

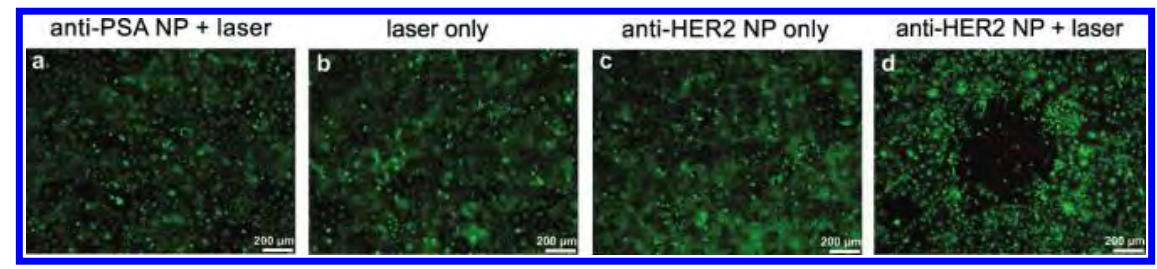

Figure 6. (a) SKOV3 cells incubated with anti-PSA conjugated branched gold nanoparticles (negative control) after laser irradiation (38 W/cm $2,5 \mathrm{~min}$ ). (b) SKOV3 cells after laser irradiation without nanoparticles. (c) SKOV3 cells with anti-HER2 conjugated nanoparticles without laser irradiation. (d) SKOV3 cells incubated with anti-HER2 conjugated branched gold nanoparticles after laser irradiation $\left(38 \mathrm{~W} / \mathrm{cm}^{2}, 5 \mathrm{~min}\right)$.

were irradiated with a $690 \mathrm{~nm}$ laser $\left(38 \mathrm{~W} / \mathrm{cm}^{2}, 5 \mathrm{~min}\right)$. After $2 \mathrm{~h}$ of incubation post-treatment, the viability of the cells was assessed using a live/dead staining. Calcein AM, a live cell stain, is a nonfluorescent dye that permeates the cell membrane and that is hydrolyzed by intracellular esterases to a green fluorescent calcein dye in live cells. Propidium iodide (PI), a dead cell staining, is membrane nonpermeable and generally excluded from viable cells. It binds to the DNA of dead cells by intrabase intercalation, generating red fluorescence. ${ }^{2,28}$ The cells stained with calcein/PI after laser irradiation are shown in Figure 5. The anti-HER2 conjugated branched gold nanoparticles were specifically bound to the SKOV3 cells. For low nanoparticle concentrations (OD 1 and 2) no cell death was observed (Figure $5 a, b)$. All cells show green fluorescence from the live cell staining. Higher nanoparticle concentrations (OD 4 and 6) produced hyperthermia induced cell death upon laser irradiation. The increased PI uptake by the dead cells within the $1 \mathrm{~mm}$ laser spot is clearly observed by red fluorescence (Figure 5c,d).

The irradiation specifications used in this experiment $\left(38 \mathrm{~W} / \mathrm{cm}^{2}, 5 \mathrm{~min}\right)$ are of the same order of magnitude as used by Hirsch et al. ( $\left.35 \mathrm{~W} / \mathrm{cm}^{2}, 7 \mathrm{~min}\right) .^{10}$ The laser power density could still be decreased by using higher nanoparticle concentrations. To induce cell death, the temperature has to be increased from $37^{\circ} \mathrm{C}$ to the hyperthermia temperature range $\left(40-47^{\circ} \mathrm{C}\right)$ according to literature. ${ }^{48}$ In contrast, earlier studies in the literature have revealed that an in vitro temperature increase of $10{ }^{\circ} \mathrm{C}$ is not sufficient to induce cell death. ${ }^{8,10}$ In vitro, a large amount of heat is dissipated to the surrounding environment so that a minimum nanoparticle temperature increase of $20^{\circ} \mathrm{C}$ is required to obtain hyperthermic cell death. Experimental temperature measurements of the branched gold nanoparticles were performed in suspension with a thermocouple upon laser irradiation $\left(38 \mathrm{~W} / \mathrm{cm}^{2}\right)$. This resulted in a temperature increase of $11^{\circ} \mathrm{C}$ for OD 1 , $16^{\circ} \mathrm{C}$ for OD $2,24^{\circ} \mathrm{C}$ for OD 4 and $28^{\circ} \mathrm{C}$ for OD 6 . At low $\mathrm{OD}$ values a fast temperature increase occurs followed by a slow saturation at higher OD values. With increasing $\mathrm{OD}$, the suspension volume absorbing the light decreases due to the higher nanoparticle density present in the suspension. As a result, the laser power decreases as a function of the path distance, causing a heating saturation. A temperature increase of more than $20^{\circ} \mathrm{C}$ was only obtained for the nanoparticle suspensions with OD 4 and 6 , which was sufficient to obtain photothermal cell death as was demonstrated above. This threshold nanoparticle concentration is only valid for our experimental conditions.

After determination of the optimal nanoparticle concentration (OD 4) to induce hyperthermic cell death, the specific character of this type of therapy was demonstrated by including the appropriate negative controls in the experiment. Hereto, the cells were incubated with the anti-PSA conjugated nanoparticles (negative control) and irradiated with the laser. In addition, the cells were also exposed to only laser irradiation without nanoparticles and to only HER2 conjugated nanoparticles without laser irradiation as a control. For the negative control, anti-PSA conjugated nanoparticles, no cell death is observed upon laser irradiation (Figure 6a). Cells irradiated with high dosages of NIR light without nanoparticles did not show any photodamage either (Figure 6b). Likewise, cells incubated with anti-HER2 conjugated nanoparticles in the absence of laser light maintained viability (Figure 6c), suggesting that neither laser light nor the nanoparticles by itself are cytotoxic. On laser exposure the anti-HER2 conjugated nanoparticle treated cells underwent photothermal destruction within the laser 
spot as determined by the viability staining (Figure $6 \mathrm{~d}$ ). A circular region of cell death is seen in the fluorescence microscopy image. The diameter of the region matches the laser spot size, confirming that cell death was confined to the laser/nanoparticle treatment area.

Although the therapeutic effect of heated branched gold nanoparticles has been proven in this work, the relationship between the generated heat and cell injury needs to be investigated in more detail. It is known from literature that the position of the nanoparticles with relation to the cell plays an important role. It has been proven that for cell internalized gold nanoparticles a higher laser power density is required to induce photothermal cell death compared to gold nanoparticles attached onto the cell membrane. ${ }^{49,50}$ Cell damage is easily achieved by disruption of the cell membrane with heated nanoparticles which focus their thermal effect into a confined area when they are accumulated on this membrane. In our experiments it is difficult to locate the nanoparticles, but the dark field images suggest that the nanoparticles are on the outer cell surface. This can explain the relatively low laser power density that was used to obtain photothermal cell death in our experiments. To have more evidence, the mechanisms of cell death due to the heat generation should be further evaluated. Not only the temperature increase itself can cause cell injury but as a consequence of this heat generation also cell membrane disruption and the subsequent influx of ions can cause cell death. ${ }^{50}$

Now that we have proven the potential of branched gold nanoparticles for photothermal therapy in vitro by determining the nanoparticle dosage and the laser power density, a transfer to in vivo tumor therapy is possible. Hereto, it is important to use branched gold nanoparticles with an absorption band tuned further into the biological window to minimize light absorption by the tissue.

\section{CONCLUSIONS}

We have successfully conjugated anti-HER2 nanobodies to branched gold nanoparticles and demonstrated their specific interaction with HER2+ SKOV3 cells. The FACS data and the dark field images clearly reveal that the anti-HER2 conjugated nanoparticles specifically bind to the cells while almost no binding was observed for the controls. The nanobodies maintained their specificity upon nanoparticle conjugation demonstrating their potential as tumor targeting ligands. Furthermore, we have achieved specific photothermal destruction of tumor cells in vitro by using two independent harmless moieties, that is, laser light and branched gold nanoparticles. Exposing the cells to either NIR light or nanoparticles did not affect cell viability. The combination of these two moieties, however, produced specific cell death confined to the laser/nanoparticle treatment area. Different nanoparticle concentrations were evaluated for this therapy and resulted in an optimal nanoparticle concentration of OD 4. Nonspecific nanoparticles conjugated with anti-PSA nanobodies did not show any cell death upon laser exposure, demonstrating the high specificity of these anti-HER2 conjugated branched gold nanoparticles.

\section{METHODS}

Materials. All materials and reagents were used as commercially obtained. Hydrogen tetrachloroaureate $\left(\mathrm{HAuCl}_{4}\right)$ and sodium citrate were purchased from Acros Organics. Bis( $p$ sulfonatophenyl) phenylphosphine dihydrate dipotassium (BSPP) was supplied by Strem Chemicals. Hydrogen peroxide $\left(\mathrm{H}_{2} \mathrm{O}_{2}\right) 30 \%(\mathrm{v} / \mathrm{v})$ was purchased from Air Products and sodium hydroxide $(\mathrm{NaOH})$ from Merck. Hydrogen chloride $(\mathrm{HCl})$ and nitric acid $\left(\mathrm{HNO}_{3}\right)$ were supplied by Honeywell. $\left(\mathrm{S}-\left(\mathrm{CH}_{2}\right)_{11}-\right.$ $\left(\mathrm{O}-\mathrm{CH}_{2}-\mathrm{CH}_{2}\right)_{6}-\mathrm{O}-\mathrm{CH}_{2}-\mathrm{CO}-\mathrm{NH}-\left(\mathrm{CH}_{2}\right)_{2}-$ maleimide) ${ }_{2}$ (maleimide-PEO-disulfide) was obtained from Prochimia. (4-(2Hydroxyethyl)-1-piperazineethanesulfonic acid (HEPES), bovine serum albumin (BSA), sodium chloride $(\mathrm{NaCl})$, magnesium chloride hexahydrate $\left(\mathrm{MgCl}_{2} \cdot 6 \mathrm{H}_{2} \mathrm{O}\right)$, calcium chloride dihydrate $\left(\mathrm{CaCl}_{2} \cdot 2 \mathrm{H}_{2} \mathrm{O}\right)$ and glucose were supplied by Sigma. Anti-HER2 (2Rb18a) and anti-PSA (N7) nanobodies were generated and produced according to standard protocols. ${ }^{33}$ Nanobodies to conjugate to maleimide-derivatized NPs are modified by replacing their N-terminal glutamine with glutamic acid and by adding residues SPSTPPTPSPSTPPC at the C-terminus, after the hexahistidine tag. Human ovarian epithelial carcinoma cells (SKOV3) and Chinese hamster ovary cells ( $\mathrm{CHO}$ ) were purchased from ATCC. RPMI 1640 cell culture medium, McCoy's 5A cell culture medium, fetal calf serum (FCS), penicillin/streptomycin (PEN/STREPT), L-glutamine, and phosphate buffered saline (PBS) were obtained from GIBCO. 24-Well plates were obtained from Nunc. Anti-His monoclonal mouse IgG1 was purchased from Serotec. Phycoerythrin (PE)-labeled rat anti-mouse IgG1,
anti-HER2 and isotype control antibodies were supplied by BD biosciences. Calcein AM and propidium iodide were obtained from Invitrogen. Potassium chloride $(\mathrm{KCl})$ was purchased from VWR.

Instrumentation. The optical properties of gold nanoparticles were characterized with UV-vis absorption spectroscopy using a Shimadzu UV-1601PC instrument with a slit width of $2 \mathrm{~nm}$ and a data interval of $0.5 \mathrm{~nm}$. Transmission electron microscopy (TEM) images of the nanoparticles were obtained using a Tecnai F30 instrument operating at $300 \mathrm{kV}$. The samples were prepared by deposition of a few droplets of the nanoparticle suspension on a carbon-coated copper grid. The gold concentration of the nanoparticles was determined with inductively coupled plasma-optical emission spectroscopy (ICP-OES) using an ICP_OES 3300 DV of Perkin-Elmer. The interaction between the nanoparticle-nanobody conjugate and the cells was analyzed with fluorescence activated cell sorting (FACS Canto Flow Cytometer (Becton Dickinson)) and darkfield microscopy (Olympus). The cells were irradiated using a home-built laser setup. In this setup a red diode laser emitting at $690 \mathrm{~nm}$ (800 mW, Opto Engine LLC) was coupled to an optical fiber with a collimating lens (74-ACR, Ocean Optics) on the other side. The laser power was adjusted using different optical density filters $(2.0,1.5,1.0,0.5)$ in a filter wheel (5213-a, Newport). A converging lens (ac254-050-b-ml, Thorlabs) was placed after the filter to focus the laser beam via a mirror onto the cells. The live and dead cells after laser illumination were imaged with a fluorescence microscope (CellR system, Olympus). 
Synthesis and Chemical Functionalization of Branched Gold Nanoparticles. Branched gold nanoparticles were prepared based on the procedure described by Hao et al. ${ }^{13} \mathrm{~A} 2 \mathrm{mg}$ portion of BSPP and $100 \mu \mathrm{L}$ of $\mathrm{H}_{2} \mathrm{O}_{2}(30 \% \mathrm{v} / \mathrm{v})$ were added to $50 \mathrm{~mL}$ of an aqueous sodium citrate solution $\left(6.8 \times 10^{-3} \mathrm{M}\right)$. Under constant stirring $100 \mu \mathrm{L}$ of $\mathrm{HAuCl}_{4}(0.075 \mathrm{M})$ was added slowly at room temperature. After $30 \mathrm{~s}$ the color of the suspension changed from colorless to blue, indicating the formation of branched $\mathrm{Au}$ nanoparticles. A $50 \mathrm{~mL}$ portion of the nanoparticle suspension was centrifuged at $4000 \mathrm{rpm}$ for $1 \mathrm{~h}$, the supernatant was discarded, and the pellet was resuspended in $10 \mathrm{~mL}$ of water.

As these branched shaped nanoparticles were not stable over time, they were stabilized by chemical functionalization with a disulfide molecule according to the method of Lin et al. ${ }^{41}$ A $2 \mathrm{~mL}$ portion of the concentrated suspension was mixed with $20 \mu \mathrm{L}$ of $\mathrm{NaOH}(0.5 \mathrm{M})$ and $200 \mu \mathrm{L}$ of the maleimide-PEO-disulfide (1.2 mM). By addition of $\mathrm{NaOH}$ the $\mathrm{pH}$ of the suspension increased to a value of 11 resulting in an enhanced electrostatic stabilization during functionalization. After $90 \mathrm{~min}$ of shaking the mixture was centrifuged at $4000 \mathrm{rpm}$ for $30 \mathrm{~min}$ and resuspended in $6 \mathrm{~mL}$ of water. The nanoparticles with a final optical density around 1 at plasmon resonance were characterized using UV-vis absorption spectroscopy and transmission electron microscopy (TEM). Inductively coupled plasma-optical emission spectroscopy (ICPOES) measurements were performed to quantify the $\mathrm{Au}$ concentration and the number of nanoparticles per milliliter. Hereto, standards were prepared from a $\mathrm{HAuCl}_{4}$ solution with concentrations between 0.1 and $2 \mathrm{ppm}$. Before measurement, different concentrations of the branched gold nanoparticles (OD $0.4,0.8,1,1.3,2$ ) and the standards were dissolved in 10 times diluted aqua regia $\left(\mathrm{HCl}: \mathrm{HNO}_{3} / 3: 1(\mathrm{v} / \mathrm{v})\right)$. With ICP-OES the gold concentration was determined (see Supporting Information) and together with the average size of the nanoparticles (determined by TEM), these data were used to calculate the number of NPs/mL.

Biofunctionalization of Branched Gold Nanoparticles with anti-HER2 and anti-PSA Nanobodies. Six milliliters of branched gold nanoparticles suspended in water with OD 1 were centrifuged for 40 min at $4000 \mathrm{rpm}$. The supernatant was removed and the particles were resuspended in $6 \mathrm{~mL}$ of HEPES buffer $(10 \mathrm{mM}, \mathrm{pH}$ 7). A $500 \mu \mathrm{L}$ aliquot of branched gold nanoparticles was mixed with $500 \mu \mathrm{L}$ of nanobodies in $1.5 \mathrm{~mL}$ eppendorf tubes. The nanobodies were diluted in HEPES buffer at different concentrations. First a broad concentration range of nanobodies $(0.1,1,10$, $100 \mu \mathrm{g} / \mathrm{mL}$ ) was used. After $1 \mathrm{~h}$ of incubation on a shaking device, the nanoparticles were centrifuged at $1500 \mathrm{rpm}$ for $90 \mathrm{~min}$. The supernatant was removed and the pellet was resuspended in $500 \mu \mathrm{L}$ of HEPES buffer. After evaluation with UV-vis absorption spectroscopy a smaller concentration range $(0.625,1.25,2.5,5,10 \mu \mathrm{g} / \mathrm{mL})$ was tested using the same protocol.

Cell Culture Maintenance. SKOV3 (HER2+) cells were maintained in RPMI 1640 cell culture medium $(500 \mathrm{~mL})$ supplemented with $10 \%$ FCS (50 mL), 100 units $/ \mathrm{mL}$ penicillin and $0.1 \mathrm{mg} / \mathrm{mL}$ streptomycin ( $5 \mathrm{~mL}$ of PEN/STREPT) and $2 \mathrm{mM}$ L-glutamine $(5 \mathrm{~mL})$. CHO (HER2-) cells were maintained in McCoy's $5 \mathrm{~A}$ medium $(500 \mathrm{~mL})$ supplemented with $10 \%$ FCS $(50 \mathrm{~mL})$, 100 units $/ \mathrm{mL}$ penicillin and $0.1 \mathrm{mg} / \mathrm{mL}$ streptomycin $(5 \mathrm{~mL}$ of PEN/STREPT) and $2 \mathrm{mM} \mathrm{L-glutamine}(5 \mathrm{~mL})$. Cells were incubated at $37{ }^{\circ} \mathrm{C}$ in a $5 \% \mathrm{CO}_{2}$ environment.

Flow Cytometric Analysis of Specific Nanoparticle Cell Interaction. Nanoparticles with anti-HER2 and anti-PSA nanobodies were prepared according to the method described above. For each nanobody, $6 \mathrm{~mL}$ of maleimide-PEO-disulfide functionalized branched gold nanoparticles suspended in HEPES buffer were mixed with $6 \mathrm{~mL}$ of nanobodies at a concentration of $5 \mu \mathrm{g} / \mathrm{mL}$ for $1 \mathrm{~h}$ in 12 aliquots of $1 \mathrm{~mL}$ each. The nanoparticles were centrifuged for $90 \mathrm{~min}$ at $1500 \mathrm{rpm}$ and the pellets were collected and resuspended in $100 \mu \mathrm{L}$ of HEPES buffer to obtain a final OD of 25. For the FACS experiment, aliquots of 400000 SKOV3 (HER2+) and CHO (HER2-) cells were incubated with $50 \mu \mathrm{L}$ of the test sample (branched gold nanoparticles with antiHER2 nanobodies) and with $50 \mu \mathrm{L}$ of the negative control (branched gold nanoparticles with anti-PSA nanobodies). As a positive control, $10 \mu \mathrm{L}$ of the anti-HER2 nanobodies $(100 \mu \mathrm{g} / \mathrm{mL})$ were added to 400000 SKOV3 and CHO cells, and, as an extra negative control, $10 \mu \mathrm{L}$ of the anti-PSA nanobodies $(100 \mu \mathrm{g} / \mathrm{mL})$ were added to the same amount of HER2 + and HER2 - cells. After $1 \mathrm{~h}$ of incubation on ice, the suspensions were washed 3 times with $2.5 \mathrm{~mL}$ PBS $(150 \mathrm{mM}, \mathrm{pH} 7.4)+0.5 \%$ BSA by centrifugation for $8 \mathrm{~min}$ at $1400 \mathrm{rpm}$. Next, the cells were incubated with $1 \mu \mathrm{L}$ of anti-His monoclonal mouse lgG1 (1 $\mu \mathrm{g} / \mu \mathrm{L}$, primary antibody) for $1 \mathrm{~h}$ on ice and again washed three times with $2.5 \mathrm{~mL}$ PBS $+0.5 \%$ BSA. In the last step, the cells were incubated with $1 \mu \mathrm{L}$ PE rat anti-mouse $\operatorname{lgG} 1(200 \mu \mathrm{g} / \mathrm{mL}$, secondary antibody) for $30 \mathrm{~min}$ on ice. After three washing steps using centrifugation, the cells were mixed for FACS analysis. As a reference, the HER2 + and HER2 - cells were only incubated with the primary and secondary antibody. After FACS analysis, all samples were characterized with dark field microscopy at $100 \times$ magnification.

Photothermal Therapy. Nanoparticles with anti-HER2 and antiPSA nanobodies were prepared according to the method described above. For each nanobody, $8 \mathrm{~mL}$ of maleimidePEO-disulfide functionalized branched gold nanoparticles, suspended in HEPES buffer, were mixed with $8 \mathrm{~mL}$ of nanobodies at a concentration of $5 \mu \mathrm{g} / \mathrm{mL}$ for $1 \mathrm{~h}$ in 48 aliquots of $1 \mathrm{~mL}$ each. The nanoparticles were centrifuged for $90 \mathrm{~min}$ at $1500 \mathrm{rpm}$, and the pellets were collected and resuspended in HEPES buffer to obtain a final OD of 12. The nanoparticles with anti-HER2 nanobodies were diluted in HEPES buffer to obtain a concentration range with ODs of $12,8,4$, and 2 . A $250 \mu \mathrm{L}$ portion of these nanoparticles was mixed with $250 \mu \mathrm{L}$ of SKOV3 cell culture medium to obtain final ODs of $6,4,2$, and 1 . The nanoparticles with anti-PSA nanobodies were diluted in HEPES to an OD of 8 and mixed with SKOV3 cell culture medium to a final OD of 4. SKOV3 (HER2+) cells were seeded in 24 well plates at 100000 cells per well and cultured overnight. The medium was removed and $500 \mu \mathrm{L}$ of nanoparticles was added to the HER $2+$ cells. After $1 \mathrm{~h}$ of incubation at $37^{\circ} \mathrm{C}$, the excess of unbound nanoparticles was removed by washing the cells three times with PBS and covering them with SKOV3 cell culture medium. The cells were exposed to laser irradiation $(690 \mathrm{~nm}$, $38 \mathrm{~W} / \mathrm{cm}^{2}, 5 \mathrm{~min}$ ) and incubated for $2 \mathrm{~h}$ at $37^{\circ} \mathrm{C}$. The cells were washed with Krebs buffer $(135 \mathrm{mM} \mathrm{NaCl}, 5.9 \mathrm{mM} \mathrm{KCl}, 1.5 \mathrm{mM}$ $\mathrm{CaCl}_{2} \cdot 2 \mathrm{H}_{2} \mathrm{O}, 1.2 \mathrm{mM} \mathrm{MgCl} \cdot 6 \mathrm{H}_{2} \mathrm{O}, 11.6 \mathrm{mM}$ HEPES, $11.5 \mathrm{mM}$ Glucose, $\mathrm{pH}$ 7.4). Cell viability after laser irradiation was assessed using a live/dead staining. Calcein AM was used as a live cell stain. The dye permeates the cell membrane and is digested to produce a green fluorescent calcein dye. Propidium iodide was used as a dead cell stain, which binds to the DNA of dead cells. A $60 \mu \mathrm{L}$ aliquot of calcein $\mathrm{AM}(1 \mathrm{mg} / \mathrm{mL})$ and $6 \mu \mathrm{L}$ of propidium iodide $(1 \mathrm{mg} / \mathrm{mL})$ were mixed with $5934 \mu \mathrm{L}$ of Krebs buffer $(\mathrm{pH}$ 7.4). A $500 \mu \mathrm{L}$ aliquot of this mixture was added to each well and incubated for $40 \mathrm{~min}$. The cells were imaged using a fluorescence microscope at $5 \times$ magnification.

Acknowledgment. Bieke Van de Broek is supported by a Ph.D. grant from the Institute for the Promotion of Innovation through Science and Technology in Flanders (IWT-Vlaanderen). The research is also funded by the European Commission (FP7, Nano3T214137). We would like to thank Olivier Richard for the TEM analysis and Kristin Coorevits for the ICP_OES measurements.

Supporting Information Available: ICP-OES measurements, nanobody coupling to branched gold nanoparticles, HER2 positive and HER2 negative expression of SKOV3 and CHO cells. This material is available free of charge via the Internet at http:// pubs.acs.org.

\section{REFERENCES AND NOTES}

1. Huang, X. H.; El-Sayed, I. H.; Qian, W.; El-Sayed, M. A. Cancer Cell Imaging and Photothermal Therapy in the NearInfrared Region by Using Gold Nanorods. J. Am. Chem. Soc. 2006, 128, 2115-2120.

2. Kirui, D. K.; Rey, D. A.; Batt, C. A. Gold Hybrid Nanoparticles for Targeted Phototherapy and Cancer Imaging. Nanotechnology 2010, 21, 105105. 
3. Jain, P. K.; El-Sayed, I. H.; El-Sayed, M. A. Au Nanoparticles Target Cancer. Nano Today 2007, 2, 18-29.

4. Weissleder, R. A Clearer Vision for in Vivo Imaging. Nat. Biotechnol. 2001, 19, 316-317.

5. Melancon, M. P.; Lu, W.; Li, C. Gold-Based Magneto/Optical Nanostructures: Challenges for in Vivo Applications in Cancer Diagnostics and Therapy. MRS Bull. 2009, 34, 415-421.

6. Au, L.; Zheng, D. S.; Zhou, F.; Li, Z. Y.; Li, X. D.; Xia, Y. N. A Quantitative Study on the Photothermal Effect of Immuno Gold Nanocages Targeted to Breast Cancer Cells. ACS Nano 2008, 2, 1645-1652.

7. Chen, J.; Wang, D.; Xi, J.; Au, L.; Siekkinen, A.; Warsen, A.; Li, Z.-Y.; Zhang, H.; Xia, Y.; Li, X. Immuno Gold Nanocages with Tailored Optical Properties for Targeted Photothermal Destruction of Cancer Cells. Nano Lett. 2007, 7, 1318-1322.

8. Dickerson, E. B.; Dreaden, E. C.; Huang, X. H.; El-Sayed, I. H.; Chu, H. H.; Pushpanketh, S.; McDonald, J. F.; El-Sayed, M. A. Gold Nanorod Assisted Near-Infrared Plasmonic Photothermal Therapy (PPTT) of Squamous Cell Carcinoma in Mice. Cancer Lett. 2008, 269, 57-66.

9. Huang, X.; Jain, P. K.; El-Sayed, I. H.; El-Sayed, M. A. Plasmonic Photothermal Therapy (PPTT) Using Gold Nanoparticles. Lasers Med. Sci. 2008, 23, 217-228.

10. Hirsch, L. R.; Stafford, R. J.; Bankson, J. A.; Sershen, S. R.; Rivera, B.; Price, R. E.; Hazle, J. D.; Halas, N. J.; West, J. L. Nanoshell-Mediated Near-Infrared Thermal Therapy of Tumors under Magnetic Resonance Guidance. Proc. Natl. Acad. Sci. U.S.A. 2003, 100, 13549-13554.

11. Gobin, A. M.; Watkins, E. M.; Quevedo, E.; Colvin, V.; West, J. L. Near-Infrared-Resonant Gold/Gold Sulfide Nanoparticles as a Photothermal Cancer Therapeutic Agent. Small 2010, 6, 745-752.

12. Day, E. S.; Bickford, L. R.; Slater, J. H.; Riggall, N. S.; Drezek, R. A.; West, J. L. Antibody-Conjugated Gold-Gold Sulfide Nanoparticles as Multifunctional Agents for Imaging and Therapy of Breast Cancer. Int. J. Nanomedicine 2010, 5, 445-454.

13. Hao, E.; Bailey, R. C.; Schatz, G. C.; Hupp, J. T.; Li, S. Y. Synthesis and Optical Properties of "Branched" Gold Nanocrystals. Nano Lett. 2004, 4, 327-330.

14. Nehl, C. L.; Liao, H. W.; Hafner, J. H. Optical Properties of Star-Shaped Gold Nanoparticles. Nano Lett. 2006, 6, 683688.

15. Baffou, G.; Quidant, R.; Girard, C. Heat Generation in Plasmonic Nanostructures: Influence of Morphology. Appl. Phys. Lett. 2009, 94, 153109.

16. Hasan, W.; Stender, C. L.; Lee, M. H.; Nehl, C. L.; Lee, J.; Odom, T. W. Tailoring the Structure of Nanopyramids for Optimal Heat Generation. Nano Lett. 2009, 9, 1555-1558.

17. Hao, F.; Nehl, C. L.; Hafner, J. H.; Nordlander, P. Plasmon Resonances of a Gold Nanostar. Nano Lett. 2007, 7, 729732.

18. Au, L.; Zhang, Q.; Cobley, C. M.; Gidding, M.; Schwartz, A. G.; Chen, J. Y.; Xia, Y. N. Quantifying the Cellular Uptake of Antibody-Conjugated Au Nanocages by Two-Photon Microscopy and Inductively Coupled Plasma Mass Spectrometry. ACS Nano 2010, 4, 35-42.

19. Gu, F. X.; Karnik, R.; Wang, A. Z.; Alexis, F.; Levy-Nissenbaum, E.; Hong, S.; Langer, R. S.; Farokhzad, O. C. Targeted Nanoparticles for Cancer Therapy. Nano Today 2007, 2, 14-21.

20. Maeda, H.; Wu, J.; Sawa, T.; Matsumura, Y.; Hori, K. Tumor Vascular Permeability and the EPR Effect in Macromolecular Therapeutics: A Review. J. Controlled Release 2000, 65, 271-284.

21. Maeda, H.; Fang, J.; Inutsuka, T.; Kitamoto, Y. Vascular Permeability Enhancement in Solid Tumor: Various Factors, Mechanisms Involved and Its Implications. Int. Immunopharmacol. 2003, 3, 319-328.

22. Peer, D.; Karp, J. M.; Hong, S.; Farokhzad, O. C.; Margalit, R.; Langer, R. Nanocarriers as an Emerging Platform for Cancer Therapy. Nat. Nanotechnol. 2007, 2, 751-760.

23. Day, E. S.; Morton, J. G.; West, J. L. Nanoparticles for Thermal Cancer Therapy. J. Biomech. Eng. 2009, 131, 074001.
24. Lowery, A. R.; Gobin, A. M.; Day, E. S.; Halas, N. J.; West, J. L. Immunonanoshells for Targeted Photothermal Ablation of Tumor Cells. Int. J. Nanomed. 2006, 1, 149-154.

25. Pils, D.; Pinter, A.; Reibenwein, J.; Alfanz, A.; Horak, P.; Schmid, B. C.; Hefler, L.; Horvat, R.; Reinthaller, A.; Zeillinger, R.; et al. In Ovarian Cancer the Prognostic Influence of HER2/Neu is not Dependent on the CXCR4/SDF-1 Signaling Pathway. Br. J. Cancer 2007, 96, 485-491.

26. Carter, P.; Presta, L.; Gorman, C. M.; Ridgway, J. B. B.; Henner, D.; Wong, W. L. T.; Rowland, A. M.; Kotts, C.; Carver, M. E.; Shepard, H. M. Humanization of an Anti-P185Her2 Antibody for Human Cancer-Therapy. Proc. Natl. Acad. Sci. U.S.A. 1992, 89, 4285-4289.

27. Carlsson, J.; Nordgren, H.; Sjostrom, J.; Wester, K.; Villman, K.; Bengtsson, N. O.; Ostenstad, B.; Lundqvist, H.; Blomqvist, C. HER2 Expression in Breast Cancer Primary Tumours and Corresponding Metastases. Original Data and Literature Review. Br. J. Cancer 2004, 90, 2344-2348.

28. Bardhan, R.; Chen, W. X.; Perez-Torres, C.; Bartels, M.; Huschka, R. M.; Zhao, L. L.; Morosan, E.; Pautler, R. G.; Joshi, A.; Halas, N. J. Nanoshells with Targeted Simultaneous Enhancement of Magnetic and Optical Imaging and Photothermal Therapeutic Response. Adv. Funct. Mater 2009, 19, 3901-3909.

29. Hellstrom, I.; Goodman, G.; Pullman, J.; Yang, Y.; Hellstrom, K. E. Overexpression of HER-2 in Ovarian Carcinomas. Cancer Res. 2001, 61, 2420-2423.

30. Berchuck, A.; Kamel, A.; Whitaker, R.; Kerns, B.; Olt, G.; Kinney, R.; Soper, J. T.; Dodge, R.; Clarkepearson, D. L.; Marks, P.; et al. Overexpression of Her-2/Neu is Associated with Poor Survival in Advanced Epithelial Ovarian-Cancer. Cancer Res. 1990, 50, 4087-4091.

31. Revets, H.; De Baetselier, P.; Muyldermans, S. Nanobodies as Novel Agents for Cancer Therapy. Expert Opin. Biol. Ther 2005, 5, 111-124.

32. Cortez-Retamozo, V.; Backmann, N.; Senter, P. D.; Wernery, U.; De Baetselier, P.; Muyldermans, S.; Revets, H. Efficient Cancer Therapy with a Nanobody-Based Conjugate. Cancer Res. 2004, 64, 2853-2857.

33. Muyldermans, S.; Baral, T. N.; Retarnozzo, V. C.; De Baetselier, P.; De Genst, E.; Kinne, J.; Leonhardt, H.; Magez, S.; Nguyen, V. K.; Revets, H; et al. Camelid Immunoglobulins and Nanobody Technology. Vet. Immunol. Immunopathol. 2009, 128, 178-183.

34. Hamers-Casterman, C.; Atarhouch, T.; Muyldermans, S.; Robinson, G.; Hamers, C.; Songa, E. B.; Bendahman, N.; Hamers, R. Naturally-Occurring Antibodies Devoid of Light-Chains. Nature 1993, 363, 446-448.

35. Edelman, G. M.; Cunningham, B. A.; Gall, W. E.; Gottlieb, P. D.; Rutishauser, U.; Waxdal, M. J. The Covalent Structure of an Entire Gamma G Immunoglobulin Molecule. J. Immunol. 2004, 173, 5335-5342.

36. Ghahroudi, M. A.; Desmyter, A.; Wyns, L.; Hamers, R.; Muyldermans, S. Selection and Identification of Single Domain Antibody Fragments from Camel Heavy-Chain Antibodies. FEBS Lett. 1997, 414, 521-526.

37. Saerens, D.; Kinne, J.; Bosmans, E.; Wernery, U.; Muyldermans, S.; Conrath, K. Single Domain Antibodies Derived from Dromedary Lymph Node and Peripheral Blood Lymphocytes Sensing Conformational Variants of ProstateSpecific Antigen. J. Biol. Chem. 2004, 279, 51965-51972.

38. Cortez-Retamozo, V.; Lauwereys, M.; Gh, G. H.; Gobert, M.; Conrath, K.; Muyldermans, S.; De Baetselier, P.; Revets, H. Efficient Tumor Targeting by Single-Domain Antibody Fragments of Camels. Int. J. Cancer 2002, 98, 456-462.

39. van der Linden, R. H. J.; Frenken, L. G. J.; de Geus, B.; Harmsen, M. M.; Ruuls, R. C.; Stok, W.; de Ron, L.; Wilson, S.; Davis, P.; Verrips, C. T. Comparison of Physical Chemical Properties of Llama VHH Antibody Fragments and Mouse Monoclonal Antibodies. BBA-Protein Struct. M. 1999, 1431, 37-46.

40. Van de Broek, B.; Frederix, F.; Bonroy, K.; Jans, H.; Jans, K.; Borghs, G.; Maes, G. Shape-Controlled Synthesis of NIR Absorbing Branched Gold Nanoparticles and Morphology 
Stabilization with Alkanethiols. Nanotechnology 2011, 22, 015601.

41. Lin, S. Y.; Tsai, Y. T.; Chen, C. C.; Lin, C. M.; Chen, C. H. TwoStep Functionalization of Neutral and Positively Charged Thiols onto Citrate-Stabilized Au Nanoparticles. J. Phys. Chem. B 2004, 108, 2134-2139.

42. Oh, E.; Susumu, K.; Blanco-Canosa, J. B.; Medintz, I. L.; Dawson, P. E.; Mattoussi, H. Preparation of Stable Maleimide-Functionalized Au Nanoparticles and Their Use in Counting Surface Ligands. Small 2010, 6, 1273-1278.

43. Hermanson, G. T. Bioconjugate Techniques; 2 nd ed.; Academic Press: San Diego, CA, 2008.

44. Giltnane, J. M.; Molinaro, A.; Cheng, H.; Robinson, A.; Turbin, D.; Gelmon, K.; Huntsman, D.; Rimm, D. L. Comparison of Quantitative Immunofluorescence with Conventional Methods for HER2/neu Testing with Respect to Response to Trastuzumab Therapy in Metastatic Breast Cancer. Arch. Pathol. Lab. Med. 2008, 132, 1635-1647.

45. Dulkeith, E.; Ringler, M.; Klar, T. A.; Feldmann, J.; Munoz Javier, A.; Parak, W. J. Gold Nanoparticles Quench Fluorescence by Phase Induced Radiative Rate Suppression. Nano Lett. 2005, 5, 585-589.

46. Kim, C. K.; Kalluru, R. R.; Singh, J. P.; Fortner, A.; Griffin, J.; Darbha, G. K.; Ray, P. C. Gold-Nanoparticle-Based Miniaturized Laser-Induced Fluorescence Probe for Specific DNA Hybridization Detection: Studies on Size-Dependent Optical Properties. Nanotechnology 2006, 17, 3085-3093.

47. El-Sayed, I. H.; Huang, X. H.; El-Sayed, M. A. Surface Plasmon Resonance Scattering and Absorption of Anti-EGFR Antibody Conjugated Gold Nanoparticles in Cancer Diagnostics: Applications in Oral Cancer. Nano Lett. 2005, 5, 829834.

48. Roti, J. L. R. Cellular Responses to Hyperthermia $\left(40-46^{\circ} \mathrm{C}\right)$ : Cell Killing and Molecular Events. Int. J. Hyperther. 2008, 24, 3-15.

49. Chen, C.-L.; Kuo, L.-R.; Chang, C.-L.; Hwu, Y.-K.; Huang, C.-K.; Lee, S.-Y.; Chen, K.; Lin, S.-J.; Huang, J.-D.; Chen, Y.-Y. In Situ Real-Time Investigation of Cancer Cell Photothermolysis Mediated by Excited Gold Nanorod Surface Plasmons. Biomater. 2010, 31, 4104-4112.

50. Tong, L.; Zhao, Y.; Huff, T. B.; Hansen, M. N.; Wei, A.; Cheng, J.-X. Gold Nanorods Mediate Tumor Cell Death by Comprising Membrane Integrity. Adv. Mater. 2007, 19, 31363141. 